\title{
Modelling of silica film growth by chemical vapour deposition: Influence of the interface properties
}

\author{
L. Vázquez, F. Ojeda, R. Cuerno ${ }^{1}$, R. Salvarezza ${ }^{2}$ and J.M. Albella \\ Instituto de Ciencia de Materiales de Madrid, CSIC, Cantoblanco, 28049 Madrid, Spain \\ ${ }^{1}$ Departamento de Matemáticas y Grupo Interdisciplinar de Sistemas Complicados, \\ Universidad Carlos III de Madrid, Av. Universidad 30, 28911 Leganés, Spain \\ 2 INIFTA, Sucursal 4, Casilla de Correo 16, 1900 La Plata, Argentina
}

\begin{abstract}
We have studied the main physical mechanisms involved in the growth of Chemical Vapor Deposition (CVD) systems. We have characterized $\mathrm{W}$ films by Scanning Tunneling Microscopy, and $\mathrm{SiO}_{2}$ films by Atomic Force Microscopy (AFM) and Infrared and Raman spectroscopies. Tungsten CVD films display an unstable growth mode since the surface roughness increases continuously with deposition time. In order to assess the physical origin of the instability we have grown silica films in a low-pressure CVD reactor from $\mathrm{SiH}_{4} / \mathrm{O}_{2}$ mixtures at $0.3 \mathrm{~nm} / \mathrm{s}$ at low $(611 \mathrm{~K})$ and high $(723 \mathrm{~K})$ temperatures. Silica films deposited at high temperature are rougher than those grown at low temperature. Moreover, they become asymptotically stable in contrast to those deposited at low temperature which are unstable. These different behaviors are explained within the framework of the dynamic scaling theory by the interplay for each growth condition between surface diffusion relaxation processes, shadowing effects, lateral growth, short-range memory effects and the relative concentration of active sites, mainly $\mathrm{SiH}$ and strained siloxane groups, and passive sites. A continuum growth equation taking into account these effects is proposed to explain the observed growth behavior for both sets of films. Computer simulations of this equation reproduce the experimental behavior.
\end{abstract}

\section{INTRODUCTION}

Many of the most important properties of thin films are related to the film surface morphology [1]. Thus, the knowledge, at a fundamental level, of the processes involved in thin film growth that finally determine the film structure could be important in order to achieve a better control of the final film quality. The necessity to achieve such control, particularly in the submicrometer range, is driven by the progressive miniaturization of solid-state devices. The development of scanning probe microscopies makes possible the routine evaluation of the film surface morphology at the nanometer-micrometer scales. These high-resolution data can then be contrasted with those predicted theoretically. These studies are also needed in those deposition techniques with important technological applications. Chemical Vapor Deposition (CVD) is one of the most widely used, partially because of its ability to grow, under certain deposition conditions, uniform deposits on complicated substrate morphologies. This property, known as conformal growth [2], indicates that, in some cases, CVD growth is governed by some stabilizing mechanism that promotes lateral rather than vertical (unstable) growth. However, as some simulations of conformal growth show [3-5], surface roughness also develops at shorter length scales. Once more, the reduction of device dimensions stresses the importance of the control of step coverage of thin films deposited in ever-smaller structures. It is clear that in order to achieve this growth control one needs to know the growth mechanisms that determine the film morphology at different length and time scales. For this reason we have addressed the study of the growth evolution in two particular systems of technological interest: tungsten and silica films grown by Low Pressure Chemical Vapor Deposition (LPCVD). 


\subsection{Theoretical concepts:}

Usually models of CVD growth [6-11] predict that the CVD film interface becomes unstable for gas diffusion controlled growth conditions. These models are purely theoretical approaches to CVD growth dynamics. Our approach is different since we first characterize a specific experimental system in order to model the CVD growth evolution. Specifically, we characterize experimentally the film surface morphology by Scanning Probe Microscopies a wide range of length and temporal scales in order to analyze the dynamic behavior of the growing interface.

\section{I.1.1 Dynamic scaling theory}

We analyze these data within the framework of the dynamic scaling theory (DST) [12-14], which allows us to identify the main growth mechanisms operating during CVD growth. DST predicts that a characteristic lateral correlation length of the system, $\xi$, obeys: $\xi(t) \propto t^{1 / z}$ where $1 / z$ is known as the coarsening exponent. The parameter most commonly employed to characterize statistically the surface morphology for a given growing system with size $L$ and observed at time $t$ is the roughness, $\sigma(L, t)$, also known as the interface width. This magnitude is defined as the mean square deviation of the local height with respect to the mean height. DST also predicts [12]: $\sigma(\mathrm{L}, \mathrm{t}) \propto \mathrm{t}^{\beta}$ for $\mathrm{L}>\mathrm{t}^{1 / 2}$ and $\sigma(\mathrm{L}, \mathrm{t}) \propto \mathrm{L}^{\alpha}$ for $\mathrm{t}^{1 / z}>\mathrm{L}$ where the exponents $\alpha$ and $\beta$ are known as the roughness and growth exponents, respectively. These three exponents are related through the expression $z=\alpha / \beta$. It is straightforward to obtain the value of the exponents $\beta$ and $1 / z$ from the AFM data since this merely requires measuring the change with deposition time of $\sigma$ and $\xi$, which are obtained directly from the AFM images. Regarding the estimation of the value of exponent $\alpha$, there are several approaches, namely it can be obtained either from the change of $\sigma$ with the sampling length [15] or from the Power Spectral Density (PSD) of the AFM images [16,17]. It can be shown that the PSD curve of a rough surface, which is a function of wavevector (inverse length) $\mathrm{k}=1 / \mathrm{L}$, obeys the following relationship: $\operatorname{PSD}(\mathrm{k}) \propto \mathrm{k}^{-(2 \alpha+2)}$. This expression implies that the $\alpha$ value can be obtained from the slope of the straight regions observed in a logarithmic plot of PSD versus $k$. Likewise, the values of $\beta$ and $1 / z$ correspond to the slopes of the straight regions of the logarithmic plots of $\sigma$ and $\xi$ versus time. The values thus obtained for the exponents can then be compared to those predicted by the different models.

\section{I.I.2 Continuum growth models}

Continuum growth models allow the simulation of the growth process by numerically integrating a differential equation that describes the local surface evolution, that is, the change with time of the surface height $h(r, t)$. The most general form of this kind of growth equation is $[13,14]$ :

$$
\partial \mathrm{h} / \partial \mathrm{t}=\mathrm{F}[\mathbf{r}, \mathrm{h}(\mathbf{r}, \mathrm{t}), \mathrm{t}]+\eta
$$

where the function $F[r, h(r, t), t]$ accounts for the different growth mechanisms, either local or non-local, which govern the morphological evolution of the system, and $\eta$ is a noise term. From the simulation of equation (1) one can compute the temporal evolution of the height profiles, the average height, interface width, PSD and the average slope. Therefore, one can derive the values of the exponents $\alpha, \beta$ and $1 / z$. It is important to note that the specific values of these exponents are dependent on substrate dimension. Although the degree of complexity of the growth equation can be rather high, it is useful to introduce briefly the simplest fundamental equations involved in this work:

a) Random deposition: In real systems the incoming particles arrive randomly, both in space and time, to the growing interface, implying the existence of local fluctuations on the surface. This fact is described by the noise term $\eta$, which is one of the main contributions to the development of the film surface roughness. Usually, these fluctuations are uncorrelated both in space and time. However, in some cases the fluctuations can present a certain degree of correlation. The systems governed only by these fluctuations, known as random deposition systems [12], are described by the following equation [18]: 


$$
\partial \mathbf{h} / \partial \mathrm{t}=\eta(\mathbf{r}, \mathbf{t})
$$

and they are characterized by an exponent $\beta=0.5$ independently of the system dimensionality $[12,18]$. b) Surface Diffusion: This is one of the main processes smoothening the surface. It can be shown that it depends on the local surface curvature and is thermally driven $[13,14,19,20]$. The simplest growth equation, usually known as the linear MBE equation, that takes into account surface diffusion and local fluctuations effects reads

$$
\partial h / \partial t=-\kappa \nabla^{4} h(r, t)+\eta(\mathbf{r}, t)
$$

where $\mathrm{k}$ is a positive constant proportional to the surface diffusion coefficient. The exponents predicted by this equation for $2+1$ dimensions are $\alpha_{\mathrm{MBE}}=1, \beta_{\mathrm{MBE}}=1 / 4$ and $1 / \mathrm{z}_{\mathrm{MBE}}=0.25[14]$.

c) Kardar-Parisi-Zhang (lateral growth, KPZ): Many growth systems show a certain tendency to grow locally at a constant velocity along the direction normal to the surface. This feature is known as lateral growth. Kardar, Parisi and Zhang [21] proposed the following equation for this type of growth:

$$
\partial \mathrm{h} / \partial \mathrm{t}=\nu \nabla^{2} \mathrm{~h}(\mathbf{r}, \mathrm{t})+(\lambda / 2)(\nabla h(\mathbf{r}, \mathrm{t}))^{2}+\eta(\mathbf{r}, \mathrm{t})
$$

The term proportional to $v$ describes evaporation/condensation (Gibbs-Thompson) effects, and that proportional to $\lambda$ describes growth along the normal direction. In $2+1$ dimensions, eq. (4) predicts, in the asymptotic limit, $\alpha_{\mathrm{KPZ}}=0.4, \beta_{\mathrm{KPZ}}=0.25$ and $\mathrm{z}_{\mathrm{KPZ}}=0.625[21]$.

In real systems, growth proceeds usually under the simultaneous action of various growth mechanisms. Hence, it is very common to observe different growth regimes, which are characterized by a different set of exponents $\alpha, \beta$ and $z$, and are separated by crossovers.

\section{EXPERIMENTAL}

The first system consists in the deposition of $\mathrm{W}$ films on $\mathrm{Si}(100)$ substrates. These films were synthesized in a SPECTRUM model 211, cold wall, single wafer, cassette-to-cassette, fully automated CVD reactor. The precursor gases were $\mathrm{WF}_{6}$ and $\mathrm{H}_{2}$. Two deposition temperatures were studied, $623 \mathrm{~K}$ (growth rate $0.35 \mathrm{~nm} / \mathrm{s}$ ) and $823 \mathrm{~K}$ (growth rate $3 \mathrm{~nm} / \mathrm{s}$ ). The deposition time spanned from 1 minute up to 8 hours. The $\mathrm{W}$ films were polycrystalline with random orientation as confirmed by $\mathrm{X}$-ray diffraction. The $\mathrm{W}$ film topography was measured with a Nanoscope III STM operating in air by using different Pt-Ir tips.

The second system is the growth of amorphous $\mathrm{SiO}_{2}$ films by Low Pressure CVD on $\mathrm{Si}(100)$ substrates also at two different temperatures, $611 \mathrm{~K}$ and $723 \mathrm{~K}$. The depositions took place in a hot wall, horizontal, low pressure tubular CVD reactor. The precursor gases were silane (diluted at $2 \%$ in nitrogen, $99.999 \%$ purity) and oxygen ( $99.9992 \%$ purity), with an oxygen/silane rate of 20 and a chamber pressure equal to 1.4 Torr. The total gas flow rate was equal to 50 and $250 \mathrm{sccm}$ for the $611 \mathrm{~K}$ and $723 \mathrm{~K}$ samples, respectively. In this case the growth rate was the same for both conditions and equal to $0.33 \mathrm{~nm} / \mathrm{s}$. We have deposited films from 10 minutes up to extremely and unusually long times (i.e., 2 days). The film morphology was characterized by a Nanoscope III Atomic Force Microscope (AFM) operating in the tapping mode at ambient conditions with silicon cantilevers.

\section{RESULTS}

\subsection{Tungsten CVD system}

Figures 1 a,b show typical STM images of a W film deposited at $623 \mathrm{~K}$ for 10 minutes. Both the roughening and the coarsening processes of the film surface can be clearly appreciated. The surface 
(a)

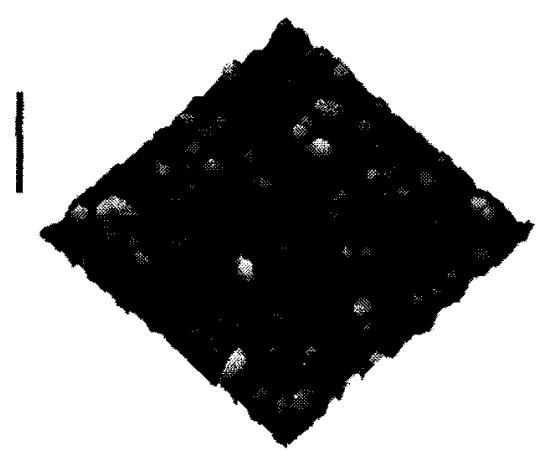

(c)

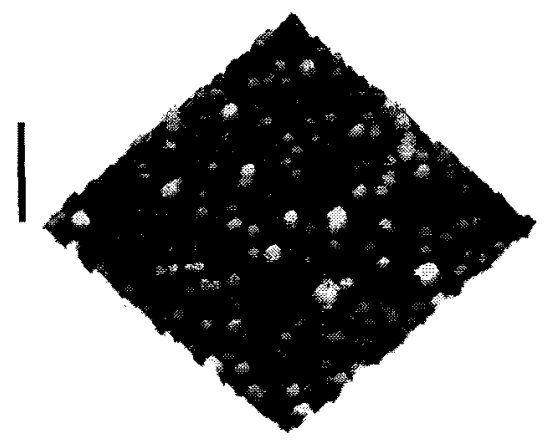

(b)

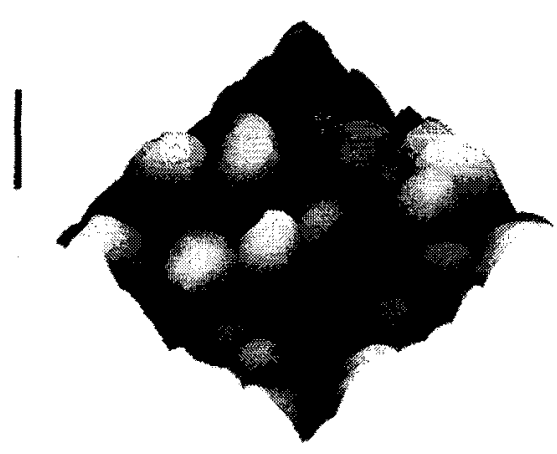

(d)

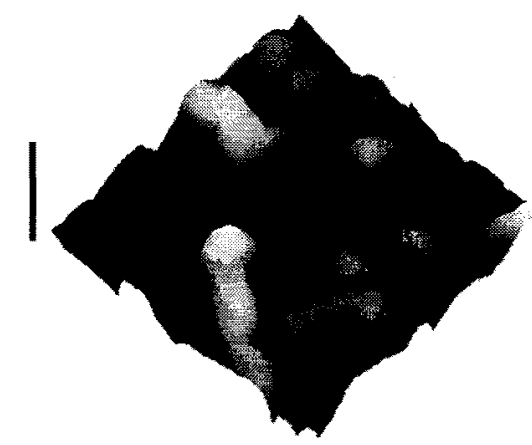

Figure 1: Three dimensional (a) $4 \times 4 \mu \mathrm{m}^{2}$ STM image of W films deposited at $623 \mathrm{~K}$ for 10 min.; (b) $4 \times 4 \mu \mathrm{m}^{2} \mathrm{STM}$ image of $W$ films deposited at $623 \mathrm{~K}$ for $400 \mathrm{~min}$.; (c) $8 \times 8 \mu \mathrm{m}^{2} \mathrm{STM}$ image of W films deposited at $823 \mathrm{~K}$ for $1.2 \mathrm{~min}$.; (d) $8 \times 8$ $\mu \mathrm{m}^{2}$ STM image of $W$ films deposited at $823 \mathrm{~K}$ for $30 \mathrm{~min}$. The bars indicate $1.14 \mu \mathrm{m}(\mathrm{a}, \mathrm{b})$ and $3.4 \mu \mathrm{m}(\mathrm{c}, \mathrm{d})$, respectively.

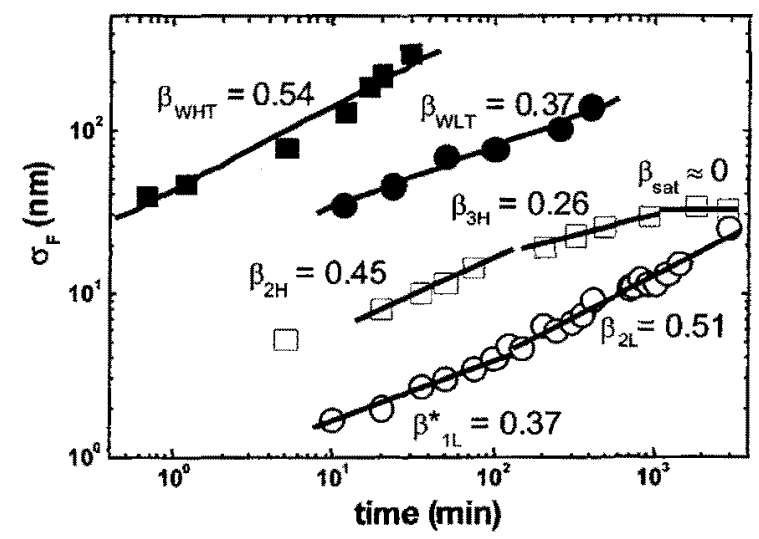

Figure 2: Logarithmic plot of the film surface roughness evolution for the $623 \mathrm{~K}(\mathbf{0})$ and $823 \mathrm{~K}(\bullet) \mathrm{W}$ films and for the $611 \mathrm{~K}$ (D) and $723 \mathrm{~K}(\mathrm{O})$ silicon dioxide films. The corresponding $\beta$ values are indicated. 
(a)

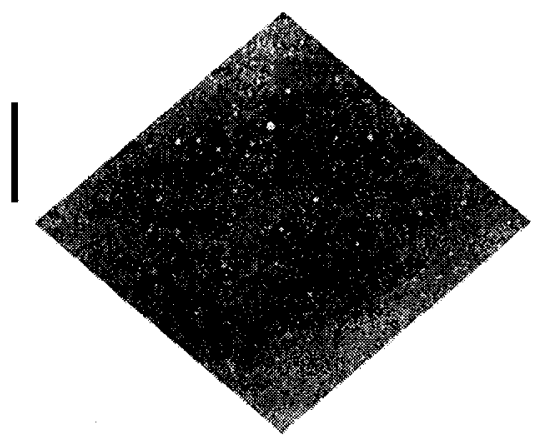

(c)

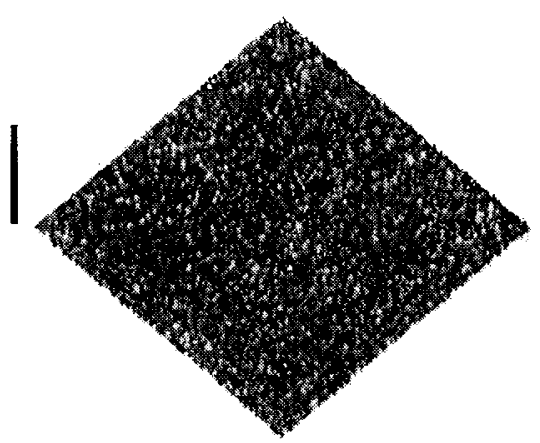

(b)

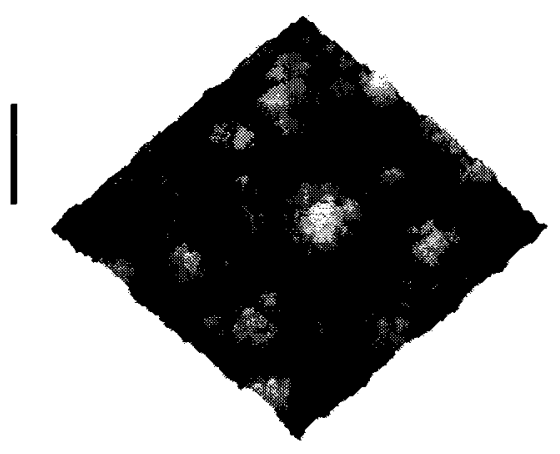

(d)

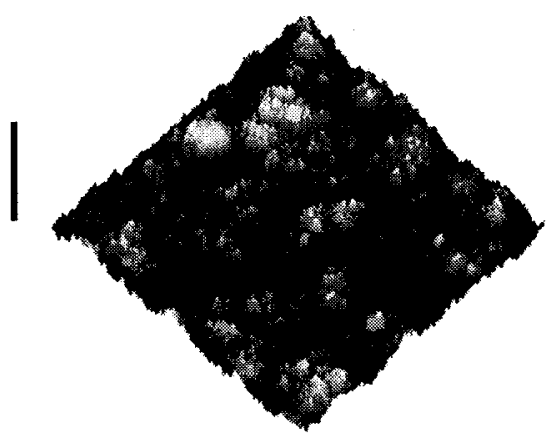

Figure 3: Three dimensional $14 \times 14 \mu \mathrm{m}^{2}$ AFM images of silicon dioxide films deposited: (a) at $611 \mathrm{~K}$ for $35 \mathrm{~min}$.; (b) at 611 $\mathrm{K}$ for 2 days; (c) at $723 \mathrm{~K}$ for $35 \mathrm{~min}$.; (d) at $723 \mathrm{~K}$ for 2 days. The bars indicate $680 \mathrm{~nm}$.
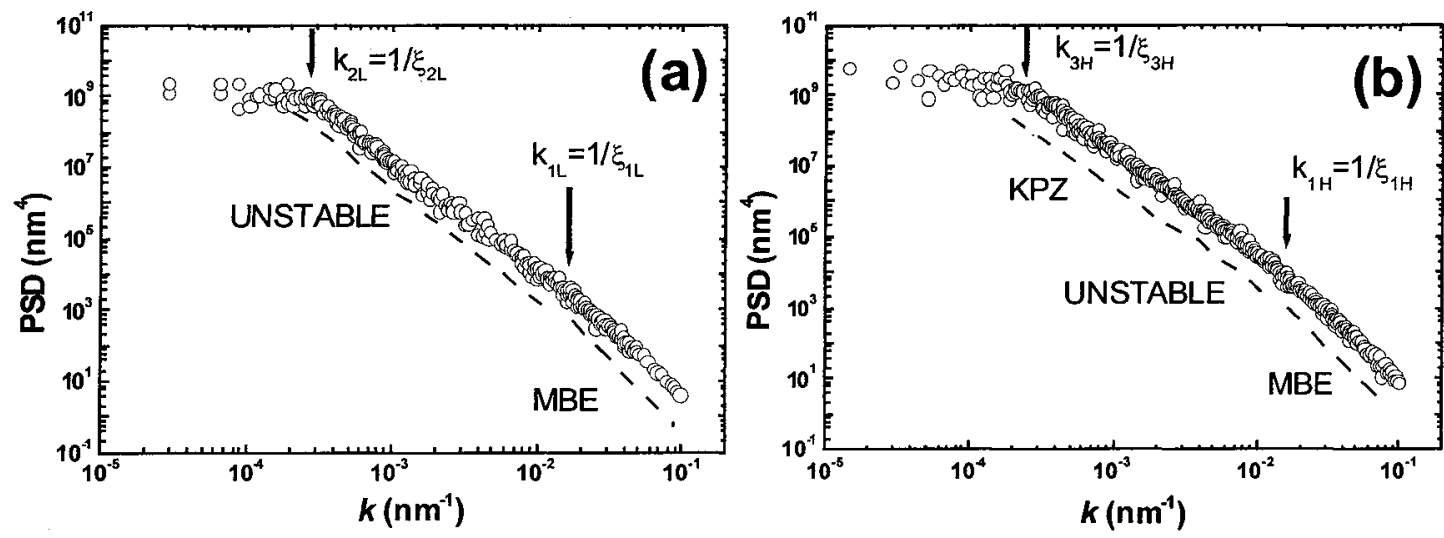

Figure 4: PSD functions of (a) the $611 \mathrm{~K}$ silicon dioxide films deposited for 48 hours; (b) the $723 \mathrm{~K}$ silicon dioxide films deposited for 48 hours. The different growth regimes are indicated for each growth temperature together with the most relevant correlation lengths. 
consists of granular structures with sharp profiles. A similar behavior, even more evident, is observed for the $\mathrm{W}$ films grown at $823 \cdot \mathrm{K}$ (figures $1 \mathrm{c}, \mathrm{d}$ ).

We plot the change of the surface roughness with deposition time for both films in figure 2 . The reported values of the exponent $\beta$ were $\beta=0.37 \pm 0.09$ for the films deposited at $623 \mathrm{~K}$ and $\beta=0.54 \pm$

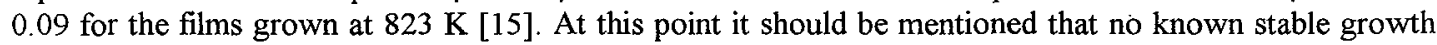
model predicts $\beta$ values larger than 0.25 in $2+1$ dimensions [14]. Therefore, these values suggest that the growth is unstable. However, the origin of the instability remains unclear. Two main causes can be considered. The first one is the shadowing effect that takes place during the CVD growth. It is well known that in CVD the reactant molecules diffuse trough the stagnant layer following random walks. Therefore, the incoming particles will impinge preferentially protruding surface regions rather than depressed surface zones. The formers shadow the latter. This process leads to competitive growth, in which the growth rate is smaller for the shadowed regions leading to an instability. This explanation would be, in principle, more suitable for the $\mathrm{W}$ films grown at $823 \mathrm{~K}$ since at this temperature the system is in the mass transportlimited regime. In this regime the slower process is the supply of reactants to the growing film interface. However, this is not the case for the $\mathrm{W}$ films deposited at $623 \mathrm{~K}$, for which the growth is in the surface kinetic-limited regime. This fact supports the consideration of the second possibility, which is related to the existence of energetic barriers at step edges on the growing $W$ films. This effect, which could explain $\beta$ values close to 0.5 , is well established for homoepitaxial singlecrystalline metal films [13]. However, its possible role in polycrystalline systems such as CVD W films has not been elucidated thus far.

In order to assess the source of the instability the most natural procedure was to rule out one of these two mechanisms by choosing an adequate system. Because of the very nature of CVD processes, it is not possible, in principle, to avoid completely shadowing effects but, on the contrary, possible step-edge barrier effects can indeed be avoided intentionally by choosing an amorphous material to be grown [16]. Thus, amorphous silica films were deposited at $611 \mathrm{~K}$ and $723 \mathrm{~K}$, and studied by AFM.

\subsection{Silicon oxide CVD system}

Figures 3 a-d display typical three dimensional AFM images of both sets of films for two deposition times, 35 minutes $(a, b)$ and 2 days (c,d). It is evident that the film surface roughens as growth time proceeds, an effect that seems to be more pronounced for those films grown at higher temperature. In the case of the low temperature films a "cauliflower-like" morphology develops, which can be 2-3 microns wide for the longest deposition times, and that determines the surface roughness at large length scales. However, for the high temperature films this structure does not appear although structures some microns wide are also observed. For both sets of films a smaller granular structure is observed. For the $611 \mathrm{~K}$ films this granular structure has a size which increases from $40 \mathrm{~nm}$ (after 20 minutes of deposition) to reach a constant value close to $80 \mathrm{~nm}$ for 3 hours of deposition. In the case of films grown at $723 \mathrm{~K}$ this structure increases in size from $35 \mathrm{~nm}(20 \mathrm{~min}$.) to a constant value close to $50 \mathrm{~nm}$ (50 minutes).

Figure 2 shows, in a log-log plot, the film surface roughness evolution, obtained directly by the AFM software, for the $611 \mathrm{~K}$ and $723 \mathrm{~K}$ films. In this figure it can be observed that, for the same deposition time, the high temperature films are always rougher than the $611 \mathrm{~K}$ films. Also, it is clear that the surface film roughness finally saturates for the $723 \mathrm{~K}$ films while for the $611 \mathrm{~K}$ films it keeps increasing continuously. Note that the surface roughness of the silica films is considerably smaller than that of the $W$ films (compare, for instance, the vertical bars of the three-dimensional AFM images in figures 1 and 3). In particular, it is important to stress the difference with the $\mathrm{W}$ films grown at $623 \mathrm{~K}$ on the same substrate and at a growth rate similar to those employed for the silica films. The analysis of the temporal dependence of the surface roughness for the silica films grown at $611 \mathrm{~K}$ suggests the existence of two growth regimes. The first one, for $10 \mathrm{~min} .<\mathrm{t}<150 \mathrm{~min}$, with $\beta^{*}{ }_{1 \mathrm{~L}}=0.37 \pm 0.03$ and the second one, for $150 \mathrm{~min} .<\mathrm{t}<2$ days, with $\beta_{2 \mathrm{~L}}=0.51 \pm 0.03$. For the silica films grown at $723 \mathrm{~K}$ figure 2 suggests the existence of two regimes. The first one, for $10 \mathrm{~min} .<\mathrm{t}<75-100 \mathrm{~min}$, with $\beta_{2 \mathrm{H}}=0.45 \pm 0.04$ and the second one, for 75 100 min. $<\mathrm{t}<15$ hours, with $\beta_{3 \mathrm{H}}=0.26 \pm 0.03$. Finally, an additional saturation regime $(\beta \approx 0)$ for 15 hours $<t<2$ days is observed. 
These data imply that for the $611 \mathrm{~K}$ samples the film growth evolution is unstable (i.e. $\beta \approx 0.5$ ) whereas for the $723 \mathrm{~K}$ films it reaches a saturation regime after an intermediate stable growth regime characterized by a relatively low value $\beta_{3 \mathrm{H}}=0.26$.

In order to perform a detailed analysis of the different growth regimes present for both deposition temperatures, it is necessary to analyze in detail both the PSD curves and the temporal change of the different correlation lengths of the systems. Since this analysis has been described in detail recently [16] we will give here the final results together with the most relevant PSD data.

\subsubsection{Growth regimes for the silica films deposited at $611 \mathrm{~K}$}

We obtain for the $611 \mathrm{~K}$ films two different growth regimes:

a) Regime I: It is characterized by $\alpha_{\mathrm{IL}}=1.0 \pm 0.1, \beta_{\mathrm{IL}}=0.25 \pm 0.03$ and $1 / z_{1 \mathrm{~L}}=0.28 \pm 0.03$. It corresponds to roughness correlations up to length scales smaller than the small granular structure, which becomes frozen for deposition times larger than 150 minutes. The corresponding lateral correlation length, $\xi_{1 \mathrm{~L}}=1 / \mathrm{k}_{1 \mathrm{~L}}$, close to the small grain size, is indicated in figure $4 \mathrm{a}$ for the PSD curve of the film deposited for 2 days. Note that the initial $\beta^{*}{ }_{1 L}$ value of 0.37 , obtained directly by the AFM software, has two important contributions coming from both regions, I and II (this is the reason to label it with *). In order to evaluate properly the $\beta_{1 L}$ value it is necessary to analyze the PSD curves [22].

b) Regime II: It is characterized by $\alpha_{2 \mathrm{~L}}=0.58 \pm 0.03, \beta_{2 \mathrm{~L}}=0.51 \pm 0.03$ and $1 / \mathrm{z}_{2 \mathrm{~L}}=0.71 \pm 0.05$. It corresponds to roughness correlations at large length scales, which are related to the cauliflower structure found on the film surface. It appears after 50 minutes of deposition, with an extent continuously increasing during the whole temporal range studied, spanning for two decades in the $\mathrm{k}$ wavevector after 2 days of growth. The corresponding lateral correlation length, $\xi_{2 \mathrm{~L}}=1 / \mathrm{k}_{2 \mathrm{~L}}$, close to the size of the "cauliflowerlike" large structure, is indicated in figure 4 a for the PSD curve of the film deposited for 2 days. It can be noted that, in this case, the value of $1 / z_{2 L}$ does not correspond, within the experimental uncertainties, to the $\beta_{2 L} / \alpha_{2 L}$ ratio although it is close to it. This discrepancy is due to the fact that for an unstable growth regime the scaling exponents are not well defined and they are effective values rather than true exponents $[13,14]$.

\subsubsection{Growth regimes for the silica films deposited at $723 \mathrm{~K}$}

In this case three regions can be distinguished in the PSD curves:

a) Region I with $\alpha_{1 \mathrm{H}}=1.0 \pm 0.04, \beta_{1 \mathrm{H}}=0.28 \pm 0.09$ and $1 / \mathrm{z}_{1 \mathrm{H}}=0.3 \pm 0.1$. It is observed in the whole temporal range, although after 50 minutes of deposition it becomes frozen. It corresponds to roughness correlations up to length scales smaller than the small granular structure. The corresponding lateral correlation length, $\zeta_{\mathrm{IH}}=1 / \mathrm{k}_{\mathrm{IH}}$, which is close to the average small grain size observed in the AFM images, is indicated in figure $4 \mathrm{~b}$

b) Region II with $\alpha_{2 \mathrm{H}}=0.75 \pm 0.03$ and $\beta_{2 \mathrm{H}}=0.45 \pm 0.04$. It is observed during the whole temporal range, although it also becomes frozen after 50 minutes of growth

c) Region III with $\alpha_{3 \mathrm{H}}=0.42 \pm 0.02, \beta_{3 \mathrm{H}}=0.26 \pm 0.03$ and $1 / \mathrm{z}_{3 \mathrm{H}}=0.67 \pm 0.09$. It is observed for $\mathrm{t}>50$ minutes. The corresponding correlation length, $\zeta_{3 \mathrm{H}}=1 / \mathrm{k}_{3 \mathrm{H}}$, which is indicated in figure $4 \mathrm{~b}$, is clearly related to the large structures, some microns wide for the longest deposition times, observed on the film surface and determining the saturated film roughness.

\section{DISCUSSION}

The next step consists in physically interpreting the observed growth regimes by comparing their exponent values with those predicted by the different growth models:

a) Region I: It is characterized for both growth temperatures by $\alpha \approx 1$ and $\beta \approx 0.25$. It is restricted to scales smaller than the typical small grain size (some nanometers wide). These exponent values correspond to those of the linear MBE equation, in which the roughness is stabilized by surface diffusion processes. The value of the $z$ exponent is consistent, within the experimental errors, with the corresponding $\alpha / \beta$ ratio. 
The relationship between the granular morphology and surface diffusion processes has been reported for different systems $[23,24]$. This regime is denoted thereafter as the MBE regime.

b) Region $I I$ is characterized by $\alpha$ values in the $0.6-0.8$ range and by $\beta$ values close to 0.5 . The latter is an evidence that this region is related to an unstable growth mechanism. The existence of this region for both sets of films allows us to unambiguously identify region II as an unstable growth regime, which will be referred to as the unstable regime.

c) Region III is observed only for the high temperature set of films. The values of the exponents are consistent with those expected for the $2+1 \mathrm{KPZ}$ growth equation. Therefore, it will be referred to as the $\mathrm{KPZ}$ region.

These growth regimes are shown in figure $4 \mathrm{a}-\mathrm{b}$, in which the PSD curves corresponding to the silica films grown for 2 days at $611 \mathrm{~K}$ and $723 \mathrm{~K}$ are displayed. The different growth regimes together with the most relevant lateral correlation lengths are indicated.

Thus, the high temperature films show an asymptotic stable, KPZ, growth regime, and both the correlation length and the surface roughness saturate after 15 hours of deposition. In contrast, the asymptotic behavior of the low temperature set of films is characterized by an unstable regime leading to a continuous increase of the film roughness. Moreover, for the same deposition time and for the whole temporal range covered in this study, the $723 \mathrm{~K}$ films are rougher than the $611 \mathrm{~K}$ films.

An important issue concerning the growth mode of CVD films is the existence of both active and passive sites for the growth. Recently, it has been reported that the morphology of CVD silica films deposited by using TEOS as a precursor is largely determined by the existence of these two types of sites and by the presence of memory effects during the growth process $[25,26]$. Under these conditions the relative concentration of active and passive sites becomes determinant for the film growth. Thus, it may be useful to perform different structural and chemical analyses of both sets of films. In this regard it is important to note that it is well-established [27-31] that among the active sites for silicon dioxide growth (i.e. $\mathrm{SiH}$ bonds, $\mathrm{SiOH}$ groups and two- and three-membered siloxane rings) SiH groups and twomembered siloxane rings are the most active ones within our temperature range. Thus, IR and Raman spectroscopies analysis [16] were focused on the determination of their respective concentration in both sets of films. These analysis showed that the most active sites are solely observed for those films grown at low temperature. Moreover, although $\mathrm{SiOH}$ groups and three-membered siloxane rings are observed for both sets of films, their concentration is higher for those films grown at $611 \mathrm{~K}$. This result implies that the sticking probability, s, will be higher for the $611 \mathrm{~K}$ films than for the $723 \mathrm{~K}$ films. This result is in agreement with other studies [3] in which, for our experimental conditions, $s$ is reported to decrease with temperature from $\mathrm{s}=0.5$ at $573 \mathrm{~K}$ down to $\mathrm{s}=0.08$ at $723 \mathrm{~K}$. Thus, the growth mode is directly related to the sticking probability, $\mathbf{s}$. This fact is specially important for the observed relationship between the predominance of lateral growth (i.e., KPZ behavior) and a low sticking probability since lateral growth is the fingerprint of both conformal and KPZ growth modes. In fact, it is also known [32] that conformal growth for $\mathrm{SiO}_{2}$ films is enhanced under low sticking probability conditions (i.e., under high deposition temperature conditions) because of the reemission of species [33].

As observed above, the difference in the relative concentration of both active and passive sites is very important when memory effects are present during film growth. This could be indeed the case for our silica films deposited at $611 \mathrm{~K}$ and $723 \mathrm{~K}$ since they do present both different active/passive site concentrations and different surface morphologies. In the case of silica films grown from TEOS, in which long range memory effects were present, when the surface concentration of active sites was not too high the film morphology resulted finger-like and full of voids. However, this is not our case since such morphology is observed in none of our sets of films. Thus, it would be interesting to study the influence on the film growth mode of short-range memory effects combined with the presence of active and passive sites. Under this scenario the film growth would take place on a growing interface with active and passive sites. Both types of sites would keep their current status, either active (sticking coefficient, $s_{a}$ ) or passive (sticking coefficient, $s_{p}$ with $s_{a}>s_{p}$ ), for a characteristic time, $\tau_{a}$ and $\tau_{p}$, respectively. We have performed Monte Carlo simulations of a random deposition process with the above characteristics for two different interface configurations, with high and low surface concentration of active sites. Figure 5 shows the corresponding 
results for the dynamics of the interface roughness. From figure 5 we conclude that when short range memory effects are present and the surface concentration of active sites is lower the surface roughness increases but, and this is important, keeping the same scaling behavior $(\beta=0.5)$. We know that in our system the silica films grown at $723 \mathrm{~K}$ have a lower concentration of active sites and are rougher than those grown at $611 \mathrm{~K}$. Thus, it seems reasonable to include short-range memory effects as one of the growth mechanisms. We have taken into account this mechanism in both discrete and continuous, Monte Carlo simulations of random deposition processes on growing interfaces. These interfaces were built with concentrations of active and passive sites similar to those observed experimentally [34]. These simulations allowed us to obtain an expression for the short-range correlated noise term for both the high and low temperature silica films. It can be shown that the temporal range characteristics of the memory effects is given by $\tau_{\|}=\tau_{\mathrm{a}^{*}} \tau_{\mathrm{p}} /\left(\tau_{\mathrm{a}}+\tau_{\mathrm{p}}\right)$ [34]. The detailed description of this analysis will be reported elsewhere [34]. At this point it is worth mentioning that our goal in the simulations presented in this work has been to reproduce qualitatively rather than quantitatively the observed growth behavior. Thus, the parameters in the simulations have been chosen with this purpose in mind. This procedure is justified because the different scaling exponents account for the rate of change of various parameters (i.e. roughness, lateral correlation length) with time or length scales. Besides, the simulations are performed on a one-dimensional substrate while the actual growth process takes place on a two-dimensional substrate.

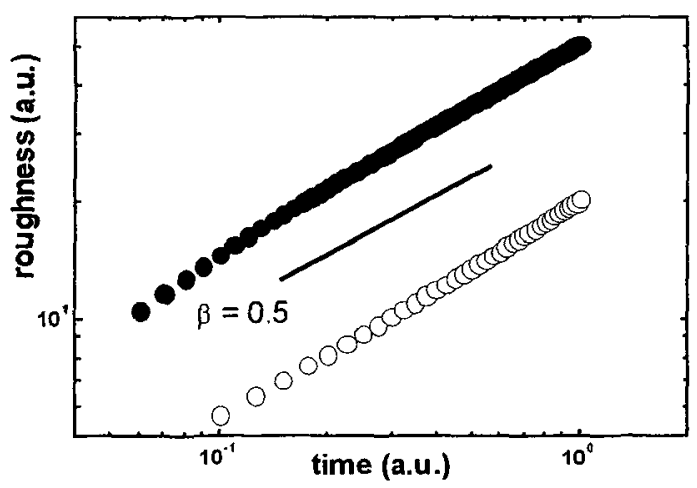

Figure 5: Logarithmic plot of the temporal evolution of the surface roughness obtained after performing a Monte Carlo simulation of the random deposition growth model for a system with high (o) and low $(\bullet)$ concentration of active sites. Note that the $\beta=0.5$ behavior expected for the random deposition model, which is indicated by the solid line, holds in both cases.

From these analysis we can draw a possible scenario for the silica film growth by CVD at $611 \mathrm{~K}$ and $723 \mathrm{~K}$, in which the main growth mechanisms are:

a) Surface diffusion regime: It determines the film growth morphology for short deposition times and short length scales. This regime appeared for both sets of films. This result suggests that the silicon oxide CVD growth equation should include a term of the type $-\kappa \nabla^{4} h(r, t)$

b) Unstable regime: As explained above, the regime for which large values of $\beta$ (close to or higher than 0.5 ) are found corresponds to unstable growth. For the low temperature films this growth mode dominates the dynamics, remaining as the asymptotic growth behavior, whereas for the high temperature set this growth mode is finally stabilized by the KPZ term. Among the various possibilities that have been considered [16] that of shadowing effects is the most likely to occur. The available models of shadowing $[35,36]$ do predict a coarsening process and large $\beta$ values, as observed in our system. In these models for a growing interface the growth velocity at a given point on the interface is roughly proportional to the local exposure angle to the gas phase $(\theta)$, and is usually expressed by the term: $\varepsilon \theta /\langle\theta\rangle$, where $\langle\theta\rangle$ represents the spatial average of $\theta$ and $\varepsilon$ is a constant $[35,36]$. 
c) KPZ (lateral growth) regime: It is observed only for the high temperature films and dominates the film growth for long deposition times. It is enhanced by the large slopes of the surface structures and originates in the relatively low surface concentration of active sites and, therefore, in the low sticking probability. The $\mathrm{KPZ}$ growth mode is represented in the growth equation by the term $(\lambda / 2)(\nabla \mathrm{h})^{2}$.

d) Noise term including short-range memory effects: It has been shown that short-range memory effects together with different active/passive sites relative concentrations can explain certain morphological differences between the $611 \mathrm{~K}$ and $723 \mathrm{~K}$ films, particularly the increased roughness observed for the 723 $\mathrm{K}$ films. As explained elsewhere [34] Monte Carlo simulations of these effects allow obtaining the expression of the short-range correlated noise term, $\eta_{\mathrm{sc}}$, for both deposition conditions.

e) It should be noted that for our system we expect the first term in eq. (4) to be negligible, since the $\mathrm{SiO}_{2}$ vapor pressure is extremely low ( $v$ is very small) in the experimental temperature range [16].

From the previous discussion we arrive at the simplest phenomenological equation for the silicon oxide CVD growth process, compatible with all the relevant physical mechanisms:

$$
\partial \mathrm{h} / \partial \mathrm{t}=-\kappa \nabla^{4} \mathrm{~h}(\mathbf{r}, \mathrm{t})+\varepsilon \theta /<\theta>+(\lambda / 2)(\nabla h(\mathbf{r}, \mathrm{t}))^{2}+\eta_{\mathrm{so}}(\mathbf{r}, \mathrm{t})
$$
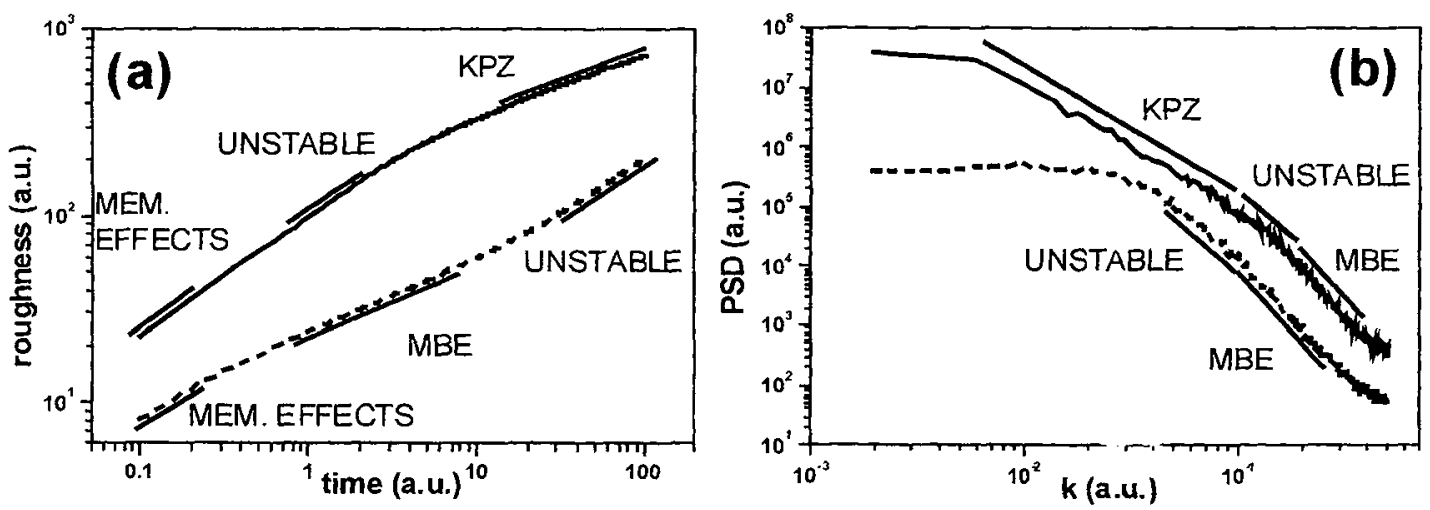

Figure 6: (a) Two logarithmic plots of the temporal evolution of the surface roughness corresponding to the height profiles generated by the proposed CVD growth equation (5) in $1+1$ dimensions for a system size of $\mathrm{L}=512$ and $\mathrm{K}=1, \varepsilon=1$ and $\Delta t$ $=0.01$. The dashed line corresponds to the low temperature memory noise with $\lambda=0.01$, and the solid line to the high temperature noise with $\lambda=0.04$. The different growth regimes are indicated. (b) Two PSD functions corresponding to height profiles generated by the proposed CVD growth equation (5) in $1+1$ dimensions for a system size of $\mathrm{L}=512$ and $\mathrm{K}=1$, $\varepsilon=\mathrm{I}$ and $\Delta t=0.01$. The dashed line corresponds to the low temperature memory noise with $\lambda=0.01$, and the solid line to the high temperature noise with $\lambda=0.04$. The different growth regimes are indicated.

In order to check the validity of eq. (5) to describe the observed growth behavior we have performed numerical simulations in $1+1$ dimensions. It should be reminded that $2+1$ simulations are limited to very small system sizes due to the large amount of calculations involved by the shadowing term [36]. Figure $6 \mathrm{a}$ shows two simulations of the roughness evolution for a system size of $L=512$, and $K=1, \varepsilon=1$ and $\Delta t=$ 0.01 , one including the low temperature memory noise (with $\lambda=0.01$, dashed line) and the other one including the high temperature noise (with $\lambda=0.04$, solid line). We recall that the numerical values of $\alpha$ and $\beta$ depend on dimensionality, and thus differ in the experiments $(2+1)$ and in our numerical simulations $(1+1)$. An unstable region with $\beta>0.5$ is observed for very short times, which corresponds to the instability originated within the temporal range characteristic of the memory effects (i.e. $t \leq 5 \tau_{\|}$). For longer times, we find for the low temperature system a regime with $\beta=0.39$. This value is close to that expected in $1+1$ dimensions for a growth process dominated by surface diffusion relaxation effects (MBE). Finally, the asymptotic regime observed displays $\beta>0.5$ indicating unstable growth due to the shadowing 
term included in eq. (5). For the high temperature system we observe that after the initial unstable region, growth is dominated by the shadowing term, which eventually yields to the KPZ $(\beta=0.33)$ growth term. Note that all these scaling behaviors, except for that found for $t \leq 5 \tau$ for both systems, are similar to those found experimentally (see figure 2) and previously reported [16]. Moreover, the roughness is larger for the high temperature set with respect to the low temperature set, again as observed experimentally (figure 2). However, the growth equation initially proposed in [16] failed to predict this effect because the noise term intensity was the same for both sets of films given that the deposition rate was the same in both cases. Finally, it is worth mentioning that the first unstable region is not observed experimentally because our shortest deposition time (a few minutes) is indeed too long compared to the extent of the short-range memory effects. Thus, whereas our former equation was essentially valid to explain the scaling behavior, eq. (5) does also explain it together with the observed roughness difference between both sets of films.

Figure $6 \mathrm{~b}$ shows the PSD curves obtained from the simulation of eq. (5) for the same three sets of conditions as figure 6a. For the low temperature condition, a first region displaying $\alpha=1.5$ is found for large $\mathrm{k}$ values, which corresponds to a regime dominated by surface diffusion relaxation processes (MBE). Also a second region with $\alpha \approx 1$ (unstable) is observed for smaller $\mathrm{k}$ values, which corresponds to the unstable region observed in figure $6 \mathrm{a}$. For the high temperature set the first region (MBE) with $\alpha=1.5$ is also observed for large $\mathrm{k}$ values. As $\mathrm{k}$ decreases a short second region with $\alpha \approx 1$ (unstable) is also observed, but for small enough $\mathrm{k}$ values a region showing $\alpha \approx 0.5$, that is KPZ behavior, appears. Thus, the same behavior found in figure $6 a$ is also observed, but in this case the initial MBE region is more clearly observed. Again a larger roughness is found for the high temperature set.

\section{CONCLUSIONS}

In order to study the main physical growth mechanisms involved in the growth of CVD films we have studied by STM and AFM the film morphology evolution and we have interpreted it within the framework of the dynamic scaling theory. First, we have studied the growth dynamics of $\mathrm{W}$ films. Our data indicate that the $\mathrm{W}$ films grown at $623 \mathrm{~K}$ or $823 \mathrm{~K}$ both show an unstable morphology (that is $\beta \geq 0.5$ ). However, the polycrystallinity of the $\mathrm{W}$ films does not allow us to identify the source of this instability. Thus, we have studied an amorphous system in which such ambiguity is not present. Therefore, we have characterized the growth evolution of silicon oxide films grown by low pressure CVD at two temperatures, $611 \mathrm{~K}$ and $723 \mathrm{~K}$, at a deposition rate of $0.33 \mathrm{~nm} / \mathrm{s}$. Deposition times as long as 2 days together with a range of AFM analysis as wide as several dozens of microns were employed in order to study the asymptotic behavior. The films were analyzed also by Raman and IR spectroscopies. From these studies, we found that the silica film is characterized first by a surface diffusion regime, which operates at short length and time scales. As time proceeds, an unstable regime appears due to the shadowing effects operating during CVD growth; these originate in the random trajectories of the precursor species towards the growing film surface. For the low temperature set of films this regime dominates the growth behavior up to 2 days of deposition. However, for the high temperature set, this unstable behavior is finally dominated by the KPZ regime that causes lateral growth of the surface structures. This regime turns out to be strongly linked to the conformal nature of CVD, which is of great importance in certain industrial applications [17]. The morphological difference between the high and low $T$ films, specifically the difference in the value of the surface roughness, is directly related to the different surface concentration of active sites (mainly Si-H and strained siloxane groups) and the different short-range memory effects for both growth conditions. For the $611 \mathrm{~K}$ films this concentration is high, implying a high sticking probability, whereas for the $723 \mathrm{~K}$ set, the concentration of reactive sites drops leading to a low sticking probability. Finally, a continuum growth equation is proposed phenomenologically to describe the silicon oxide CVD growth for a wide range of temporal and spatial scales, as well as for different experimental conditions.

\section{Acknowledgements}

This work was partially supported by the $7220-\mathrm{DE} / 082$ project from ECSC, the $07 \mathrm{M} / 0710 / 97$ project from CAM and Ministerio de Educación y Cultura (MEC, programa de Cooperación Científica con 
Iberoamérica) and the DGES project BFM2000-0006 from MEC. It was pertormed also within the framework of the CSIC/CONICET research cooperation program.

\section{References}

[1] Barna P.B. and Adamik M., in Protective Coatings and Thin Films, Pauleau Y. and Barna P.B. (Eds.) (Kluwer Academic Publishers, Dordrecht, 1997), pp. 279-297.

[2] Randall J, and Parmeter J.E., Critical Reviews in Solid State and Materials Sciences 18 (1993) $175-$ 237.

[3] Watanabe K. and Komiyama H., J. Electrochem. Soc. 137 (1990) 1222-1227.

[4] Ikegawa M. and Kobayashi J., J. Electrochem. Soc. 136 (1989) 2982-2986.

[5] Coronell D.G. and Jensen K.F., J. Electrochem. Soc. 141 (1994) 2545-2551.

[6] Van der Brekel C.H.J. and Jansen A.K., J. Cryst. Growth 43, (1978) 364-370.

[7] Jansen A.K. and Van der Brekel C.H.J., J. Cryst. Growth 43 (1978) 371-377.

[8] Palmer B.J. and Gordon R.G., Thin Solid Films 158 (1988) 313-341.

[9] Palmer B.J. and Gordon R.G., Thin Solid Films 159 (1989) 141-159.

[10] Bales G.S., Redfield A.C. and Zangwill A., Phys. Rev. Lett. 62 (1989) 776-779.

[11] Viljoen H.J., Thiart J.J. and Hlavacek V., AIChE J. 40 (1994) 1032-1045.

[12] Family F. and Vicsek T., J. Phys. $A$ 18 (1985) L75-L79.

[13] Krug J., $A d v$. Phys. 46(1997) 139-282

[14] Barabási A.L. and Stanley H. E., Fractal Concepts in Surface Growth (Cambridge University Press, Cambridge, 1995).

[15] Vázquez L., Salvarezza R.C., Albano E., Arvia A.J., Hernández-Creus A., Levy R. and Albella J.M., Chemical Vapor Deposition 4 (1998) 89-91.

[16] Ojeda F., Cuerno R., Salvarezza R. and Vázquez L., Phys. Rev. Lett. 84 (2000) 3125-3128.

[17] Ojeda F., Cuerno R., Salvarezza R. and Vázquez L., J. Phys. IV France 9 (1999) 265-271.

[18] Tong W.M. and Williams R.S., Ann. Rev. Phys. Chem. 45 (1997) 401-438.

[19] Villain J., J. Phys. I 1 (1991) 19-42.

[20] Lai Z.-W. and Das Sarma S., Phys. Rev. Lett., 66 (1991) 2348-2351

[21] Kardar M., Parisi G. and Zhang Y.C., Phys. Rev. Lett., 56 (1986) 889-892.

[22] By integrating the PSD curves from the maximum $k$ available down to $k_{0}$.

[23] Vázquez L., Salvarezza R., Herrasti P., Ocón P., Vara J.M. and Arvia A.J., Surf. Sci. 345 (1996) 17-26.

[24] Otsuka I and Iwasaki I., J. Vac. Sci. Technol. B 14 (1996) 1153-1156.

[25] Tsukamoto K., Cheng D., Komiyama H., Nishimoto Y., Tokumasu N. and Maeda K., Jpn. J. Appl. Phys., 38 (1999) L68-L70.

[26] Cheng D., Tsukamoto K., Komiyama H., Nishimoto Y., Tokumasu N. and Maeda K., J. Appl. Phys., 85 (1999) 7140-7145

[27] Kanashima T., Okuyama M. and Hamakawa Y., Jpn. J. Appl. Phys., 36 (1997) 1448-1452

[28] Sun Q., Yu J.Z., Zhou L., Li Z.Q., Tang Z., Ohno K. and Kawazoe Y., Europhys. Lett. 43 (1998) 47 52.

[29] Shelby J.E., J. Non-Cryst. Solids 179 (1994) 138-147.

[30] Nakano T., Mura N. and Tsuzumitami A., Jpn. J. Appl. Phys., 34 (1995) L1064-L 1067

[31] Ceresoli D., Bernasconi M., Iarlori S., Parrinello M. and Tosatti E., Phys. Rev. Lett., 84 (2000) 3887-3889.

[32] Cooke M.L. and Harris G., J. Vac. Sci. Tech. A 7 (1989) 3217-3221.

[33] Cheng L., McVittie J.P. and Saraswat K.C., Appl. Phys. Lett., 58 (1991) 2147-2149.

[34] Ojeda F., Cuerno R., Salvarezza R. and Vázquez L., (in preparation).

[35] Karunasiri R.P.U., Bruinsma R. and Rudnick J., Phys. Rev. Lett. 62 (1989) 788-791.

[36] Yao J.H. and Guo H., Phys. Rev. E 47 (1993) 1007-1011. 